\title{
Quantitative Minimization for Liftoff Distance Variation using Radial Basis Function Network on Eddy current Nondestructive Testing
}

\author{
Jaejoon $\mathrm{Kim}^{1}$, Daewon $\mathrm{Kim}^{2}$, Lalita Udpa ${ }^{3}$ and Satish S. Udpa ${ }^{3}$ \\ ${ }^{1}$ School of Computer and Communication, Daegu University \\ 201 Daegudae-ro, Gyeongsan, Gyeongbuk, 38453 Korea \\ jjkimisu@daegu.ac.kr \\ ${ }^{2}$ Multimedia Engineering Department of Electronics and Computer Engineering \\ College of Engineering Dankook University \\ San-29, Anseo-dong, Cheonan, Choongnam, 330-714 Korea \\ ${ }^{3}$ Department of Electrical and Computer Engineering, Michigan State University \\ East Lansing, MI, 48824 USA
}

\begin{abstract}
This paper presents a technique to evaluate liftoff characteristics on zirconium weld metal using the impedance signals collected by ferrite pot core probe. The inspection system uses sine waveform as the excitation source of a multi-line coil and captures the transient fields associated with the induced eddy currents via a sensor. The collected signals by $1 \mathrm{~mm}$ increment of distance can be divided into training and testing data in radial basis function network that was developed to estimate the accuracy for liftoff distance effect. The proposed approach of radial basis function network has been successfully developed to evaluate liftoff distance effect on test samples.
\end{abstract}

Keyword-Eddy current probe, zirconium weld metal, ferrite pot core, liftoff, RBF network

\section{INTRODUCTION}

Eddy current testing (ECT) methods have served as the primary non-destructive evaluation (NDE) technique for applications in the aviation industry, nuclear, aerospace, and other industries to examine and detect surface corrosion, flaws and other material discontinuities [1]. ECT technique has been usually considered on various parameters including the probe design and testing parameters on material characteristic applications. The impedance values of EC signal provide information about the severity of samples. However, depending on inspection environment including applied area and surface condition, lift-off distance provides information about the depth of the defect or status of surface conditions.

In Eddy current testing, liftoff effect occurs when the probe of scanning system is not contact with test surface [2]. Liftoff factor can affect to accurate and precise inspection on test materials. In general, eddy current testing needs to be in contact with test sample region. Due to any air gap between the probe and material, signal information may have false indication of a test material. In order to avoid inaccurate signal information, lots of various liftoff compensation techniques have been studied and developed to reduce sensitivity of inspection equipment [3]. Large liftoff distances will affect testing accuracy, so it is important to keep the probe in close contact with the surface during testing. In addition, the conductivity of the metal tested also affects testing frequency. The greater the conductivity of a material, the greater the flow of eddy currents on the surface. Proper selection of testing frequency for material thickness and penetration depth also has been considered on smooth or irregular surface condition.

In this paper, we proposed the application of radial basis function network analysis to estimate liftoff distance effect in eddy current inspection system. The testing results of different liftoff variations are presented. The rest of the paper is organized as follows. Section 2 presents the review of liftoff distance effect for eddy current inspection system and data collection. Section 3 discusses the radial basis function network. Section 4 presents the experimental result and the liftoff variation performance with different conductivity for the test samples and Section 5 provides some concluding remarks. 


\section{EDDY CURRENT INSPECTION SYSTEM WITH LIFTOFF EFFECT}

In Eddy current testing system, a liftoff is one of the main factors for inspection of surface or subsurface on test samples. The liftoff distance can affect to signal strength which has been shown in major information on scanning area including various kind of flaw types. Many works have been done to mitigate or estimate liftoff distance effect. He et al. [4] presented reduction of liftoff effects in pulsed eddy current system on two layered structure in time and frequency domain. Tian et al. proposed a normalization approach for compensating the liftoff variation during the PEC inspection [5]. Zhang et al. [6] investigated characteristics of lift-off and tilt variations to prevent hiding signal information caused by liftoff effect using finite element modeling which has been exploited in electromagnetic field and impedance of a cylindrical probe-coil. Mandache et al. [7] researched time gating technique for liftoff compensation in conventional and pulsed eddy current inspection.

Proper selection of probes and optimization of test parameters such as test frequency, phase angle, and gain is important. In many situations, multi-frequency methods are also adopted [8]. The Eddy current inspection system comprises a probe and impedance measurement. Fig. 1(a) shows an eddy current probe wound on a pot core with $3.38 \mathrm{~mm}$ OD and of $1.22 \mathrm{~mm}$ ID as shown in Fig. 1(b). The test samples are zirconium weld metal with different conductivity. $1 \mathrm{MHz}$ of frequency for sine waveform is used as excitation current in order to measure real and imaginary impedance on test samples.

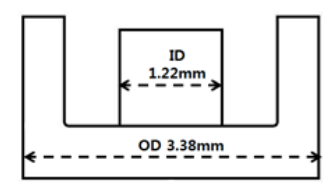

(a)

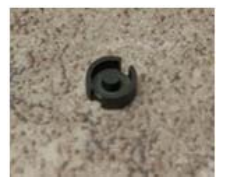

(b)

Fig. 1. The geometry of ferrite pot core

As shown in Fig. 2, a liftoff scanning system is used to generate the 1-D scan around specific location of test sample. In data collection process, $1 \mathrm{~mm}$ of liftoff distance has been used up to $10 \mathrm{~mm}$ of distance. Once data collection, a total data is divided into train and test data with 7 to 3 ratio. For training data, RBF network has been adopted and applied to test data set.

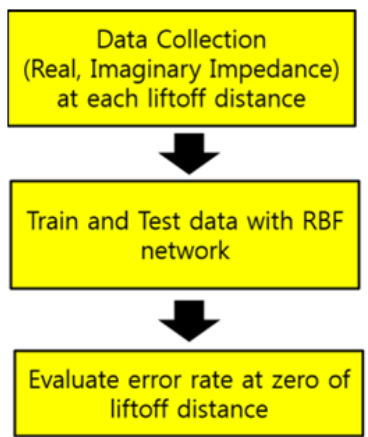

Fig. 2. The overall block diagram with a scanning system

\section{RADIAL BASIS FUNCTION NETWORK}

The idea of Radial Basis Function (RBF) Networks derives from the theory of function approximation [9]. Multi-Layer Perceptron (MLP) networks employs a hidden layer of sigmoidal units for approximating functions [10]. Unlike MLP, main feature of RBF networks is two-layer feed-forward networks with hidden nodes with a set of radial basis functions. This network training is divided into two stages: first the weights from the input to hidden layer are determined, and then the weights from the hidden to output layer.

Given a set of $N$ data points, $\mathbf{x}^{p}$ in a multi-dimensional space need to be mapped onto the corresponding target output $t^{p}$ such that

$$
f\left(\mathbf{x}^{p}\right)=t^{p} \forall p=1, \ldots, N
$$

From Eq. (1), we need to find a radial basis function which satisfices the form $\phi\left(\left\|\mathbf{x}-\mathbf{x}^{p}\right\|\right)$ where $\phi(\cdot)$ is some non-linear function and $\left\|\mathbf{x}-\mathbf{x}^{p}\right\|$ is Euclidean distance between $\mathbf{x}$ and $\mathbf{x}^{p}$. The output is then a linear combination of the basis functions, i.e. 


$$
f(\mathbf{x})=\sum_{p=1}^{N} w_{p} \phi\left(\left\|\mathbf{x}-\mathbf{x}^{p}\right\|\right)
$$

where, $w_{p}$ is a weight vector satisfying the function on data points. Finally, by combining Eq. (1) and Eq. (2), we can derive the following equation.

$$
f\left(\mathbf{x}^{q}\right)=\sum_{p=1}^{N} w_{p} \phi\left(\left\|\mathbf{x}^{q}-\mathbf{x}^{p}\right\|\right)=t^{q}
$$

Similarly, Eq (3) can be represented in following matrix form by finding the inverse of $\boldsymbol{\Phi}$ :

$$
\Phi \mathbf{w}=\mathbf{t} \text { or } \mathbf{w}=\Phi^{-1} \mathbf{t}
$$

where, the vectors $\mathbf{t}=\left[t^{p}\right]$ and $\mathbf{w}=\left[w_{p}\right]$ and matrix $\boldsymbol{\Phi}=\phi\left(\left\|\mathbf{x}^{q}-\mathbf{x}^{p}\right\|\right)$. Therefore, we can find a continuous function passing on each data point when we have the weights.

\section{EXPERIMENTAL RESULTS}

\section{A. Experimental Setup}

The quantitative liftoff variation experiment was employed to measure test specimen with zirconium weld metal. Fig. 3 shows experimental setup used with the ferrite pot core probe as shown in Figure 1. Since measuring region of sample geometry is very small, we used smaller core to reduce edge effect. The impedance values were collected from center of the sample to avoid edge effects. The conductivity of test samples is sample $1(\rho=53.72 \mu \Omega-\mathrm{cm})$ and sample $2(\rho=55.19 \mu \Omega-\mathrm{cm})$ respectively. Table I described parameter information for liftoff variation experiment. The range for applied liftoff values is 0 to $10 \mathrm{~mm}$ and collected by $1 \mathrm{~mm}$ of increment.

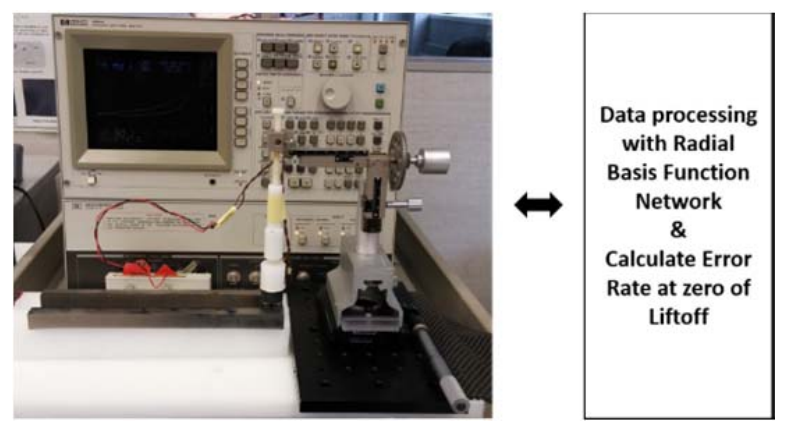

Fig. 3. The experimental scanning system

TABLE I

Parameter values for experimental scanning system

\begin{tabular}{|l|l|}
\hline Ferrite core type & Pot core shape \\
\hline Frequency & $1 \mathrm{MHz}$ \\
\hline Averaging times & 256 \\
\hline Number of coil turns & 30 \\
\hline Coil thickness & $0.1 \mathrm{~mm}$ \\
\hline
\end{tabular}

\section{B. RBF Network Results}

In our RBF analysis, the major characteristics are training and testing network. There is no general criterion for choosing the number of training and testing data. In this research, we used $70 \%$ of training data and $30 \%$ of testing data. Two different experiments were reviewed based on selection of factors for training data: One contained real and imaginary with liftoff values. The other contained real and imaginary values without liftoff values. Table II and III show results with two experiments. The error rates are not much different on both experiments. 
TABLE II

Results for training data with liftoff factor

\begin{tabular}{|c|c|c|c|c|c|c|c|}
\hline & \multirow{2}{*}{$\begin{array}{c}\text { Testing } \\
\text { liftoff }\end{array}$} & \multicolumn{2}{|c|}{ Testing Value } & \multicolumn{2}{|c|}{ Target Value } & \multicolumn{2}{|c|}{ Error Rate } \\
\hline & & real & imaginary & real & imaginary & real & imaginary \\
\hline \multirow{3}{*}{ Sample 1} & 0.2 & 3.8345 & 17.5778 & 3.97024 & 17.6095 & $0.674 \%$ & $0.674 \%$ \\
\hline & 0.5 & 2.57466 & 19.7693 & 3.97024 & 17.6095 & $0.173 \%$ & $0.173 \%$ \\
\hline & 0.8 & 1.96638 & 20.8336 & 3.97024 & 17.6095 & $-0.076 \%$ & $-0.076 \%$ \\
\hline \multirow{3}{*}{ Sample 2} & 0.2 & 3.88037 & 17.6176 & 3.97377 & 17.6387 & $0.188 \%$ & $0.188 \%$ \\
\hline & 0.5 & 2.64387 & 19.684 & 3.97377 & 17.6387 & $0.222 \%$ & $0.222 \%$ \\
\hline & 0.8 & 1.99525 & 20.7997 & 3.97377 & 17.6387 & $-0.028 \%$ & $-0.028 \%$ \\
\hline
\end{tabular}

TABLE III

Results for training data without liftoff factor

\begin{tabular}{|c|c|c|c|c|c|c|c|}
\hline & \multirow{2}{*}{$\begin{array}{c}\text { Testing } \\
\text { liftoff }\end{array}$} & \multicolumn{2}{|c|}{ Testing Value } & \multicolumn{2}{|c|}{ Target Value } & \multicolumn{2}{|c|}{ Error Rate } \\
\hline & & real & imaginary & real & imaginary & real & imaginary \\
\hline \multirow{3}{*}{ Sample 1} & 0.2 & 3.8345 & 17.5778 & 3.97024 & 17.6095 & $0.660 \%$ & $0.660 \%$ \\
\hline & 0.5 & 2.57466 & 19.7693 & 3.97024 & 17.6095 & $0.183 \%$ & $0.183 \%$ \\
\hline & 0.8 & 1.96638 & 20.8336 & 3.97024 & 17.6095 & $-0.073 \%$ & $-0.073 \%$ \\
\hline \multirow{3}{*}{ Sample 2} & 0.2 & 3.88037 & 17.6176 & 3.97377 & 17.6387 & $0.302 \%$ & $0.302 \%$ \\
\hline & 0.5 & 2.64387 & 19.684 & 3.97377 & 17.6387 & $0.153 \%$ & $0.153 \%$ \\
\hline & 0.8 & 1.99525 & 20.7997 & 3.97377 & 17.6387 & $-0.057 \%$ & $-0.057 \%$ \\
\hline
\end{tabular}

\section{CONCLUSION}

A liftoff variation characteristic with RBF network analysis has been reviewed. Eddy current testing based on RBF network features have shown promising results in estimating liftoff distance on different conductivity materials. The system is capable of estimating zero of liftoff distance from variable liftoff distances with $1 \mathrm{~mm}$ increment. The system also shows similar results based on training RBF network with liftoff factors or without liftoff factors. Later this system with liftoff variation can be useful for fast scanning on various unflatten samples including aircraft to allow much more rapid inspection over large areas. More extensive evaluating with large volume of data is currently underway.

\section{ACKNOWLEDGMENT}

This research was supported by the Daegu University Research Grant, 2015

\section{REFERENCES}

[1] Javier García-Martín, Jaime Gómez-Gil, and Ernesto Vázquez-Sánchez, "Non-Destructive Techniques Based on Eddy Current Testing," Sensors, Vol. 11, pp. 2525-2565, 2011.

[2] Leszek Dziczkowski, "Elimination of Coil Liftoff from Eddy Current Measurements of Conductivity," IEEE Transactions on Instrumentation and Measurement, Vol. 22, No. 12, pp. 3301-3307, 2013.

[3] Ricci, M., Silipigni, G., Ferrigno, L., Laracca, M., Adewale, I.D., Gui Yun Tian, "Analysis of the influence of lift-off variation on EddyCurrent images," in Proc. Metrology for Aerospace (MetroAeroSpace) 2015 IEEE, pp. 182-187.

[4] Yunze He, Mengchun Pan, Feilu Luo, and Guiyun Tian, "Reduction of Lift-Off Effects in Pulsed Eddy Current for Defect Classification," IEEE Transactions on Magnetics, Vol. 47, No. 12, pp. 4753-4760, 2011.

[5] Gui Yun Tian, Yong Li, and Catalin Mandache, "Study of Lift-Off Invariance for Pulsed Eddy-Current Signals," IEEE Transactions on Magnetics, Vol. 45, No. 1, pp. 184-191, 2009.

[6] Yu-hua Zhang, Fei-lu Luo, Hui-xian Sun, "Impedance Evaluation of a Probe-Coil's Lift-off and Tilt Effect in Eddy-Current Nondestructive Inspection by 3D Finite Element Modeling," in Proc. 17th World Conference on Nondestructive Testing, 25-28 Oct 2008, Shanghai, China.

[7] Catalin Mandache, Mike Brothers, and Vivier Lefebvre, "Time domain lift-off compensation method for eddy current testing," NDT.net, Vol. 10, No. 6, 2005. 
[8] Wuliang Yin, Philip J. Withers, Umesh Sharma, Anthony J. Peyton, "Noncontact Characterization of Carbon-Fiber-Reinforced Plastics Using Multifrequency Eddy Current Sensors," IEEE Transactions on Instrumentation and Measurement, Vol. 58, No. 3, pp. 738-742, 2009.

[9] Martin D. Buhmann, Radial Basis Functions: Theory and Implementations. Cambridge University, 2003.

[10] G.F. Luger. Artificial Intelligence. Addison Wesley, 2005. 